\title{
Melting and evaporation in classical two-dimensional clusters confined by a Coulomb potential
}

\author{
W. P. Ferreira, ${ }^{1, *}$ F. M. Peeters ${ }^{2, \dagger}$ and G. A. Farias ${ }^{1, *}$ \\ ${ }^{1}$ Departamento de Física, Universidade Federal do Ceará, Caixa Postal 6030, Campus do Pici, 60455-760 Fortaleza, Ceará, Brazil \\ ${ }^{2}$ Department of Physics (U213), University of Antwerp, Groenenborgerlaan 171, B-2020 Antwerpen, Belgium
}

(Received 9 June 2005; published 6 October 2005)

\begin{abstract}
The thermal properties of a two-dimensional classical cluster of negatively charged particles bound by a punctual positive charge are presented. The melting phenomenon is analyzed and the features which characterize such a solid-liquid transition are highlighted. We found that the presence of metastable states strongly modifies the melting scenario, and that the melting temperature of the system is determined by the height of the saddle point energy separating the ground state and the metastable state. Due to the particular type of confinement potential considered in this paper, we also found that, at sufficiently large temperature, the cluster can become thermally ionized.
\end{abstract}

DOI: 10.1103/PhysRevE.72.041502

PACS number(s): 83.10.Tv, 36.40.Ei, 64.90.+b

\section{INTRODUCTION}

There has been an increased interest in the properties of two-dimensional (2D) clusters of charged or neutral particles. These 2D clusters are remarkable systems with nontrivial and unexpected physical behavior. One of the reasons for this increased interest is that such systems are models for many different experimental realizable systems. Surface electrons on a thin liquid helium film $[1,2]$, colloidal suspensions on an inert substrate [3], dusty plasmas [4], and electrons confined in low-dimensional semiconductor structures [5] are some experimental realizations of such systems. Even several model systems were recently realized experimentally consisting of macroscopic objects as charged metallic balls [6] and magnetic disks [7,8]. In addition, 2D clusters serve as very convenient systems to test theoretical predictions for different physical phenomena. For example, we can cite the crystallization of a 2D electron gas in a triangular lattice predicted by Wigner [9] and the microscopic theory of Kosterlitz-Thouless-Halperin-Nelson-Young for phase transition in a 2D system [10-12], which is very different from the analog 3D system.

Most of the papers dealing with 2D systems describe the behavior of large systems, where the boundaries are not so important. In such cases, the effect of the surface on most of their thermodynamic properties is negligibly small when compared with the effects produced by the total system. In the opposite limit, small clusters are strongly affected by the boundaries. Recently, some experiments [13-15] and numerical simulations [16-19] investigated the structural and dynamical properties of small 2D clusters. Such studies clearly emphasize the importance of surfaces and finite size effects. For example, the formation of a shell structure in clusters with a small number of particles, the melting phenomenon which is characterized by a two step process [19], the stability against intershell rotation which is observed in clusters with a "magic" number of particles [20], and the

\footnotetext{
*Electronic address: wandemberg@ fisica.ufc.br

†Electronic address: francois.peeters@ua.ac.be
}

splitting of frequencies in the presence of a perpendicular magnetic field which is associated with the edge magnetoplasmons, and which is not observed in infinite systems $[18,21,22]$.

The melting phenomenon in three-dimensional (3D) crystals is significantly different from the melting in twodimensional (2D) crystals. In the latter case, and as pointed out by several authors, the transition from a solid phase to a complete isotropic liquid phase can be characterized by a two-step process with an intermediary hexatic phase [10-12]. However, such a melting scenario is not unique and it depends on the considered system. Furthermore, melting of 2D clusters is nonuniversal $[15,16]$.

The melting phenomenon in small clusters of charged particles has also received considerable attention in recent years $[14,19,23-25]$. Very interesting properties have been observed in such systems, such as, e.g., a reentrant melting behavior [24]. Recently, we proposed a different type of classical atom which is bound not by a parabolic or hard wall type of confinement potential, but a Coulomb-type of potential [26]. In this system, the electrons are confined by a punctual positive charge positioned under the 2D plane. Earlier, we showed that, as a function of the strength of such a confinement potential, symmetry broken configurations can appear as a ground state, and in addition particles can unbound, i.e., evaporate [26]. It is worth commenting that the parabolic confinement potential is equivalent to a uniform neutralizing background, which is essentially different from our Coulomb confined case. The Coulomb type of confinement of the present system offers two more important properties when compared to the parabolic case: (1) the possibility to tune the strength of the confinement potential, which allows us to explore the importance of this feature in the behavior of the static and dynamic properties of the system, and (2) the particles require only a finite amount of energy to move to infinity, i.e., particles can evaporate. The present system is not purely academic, but may be realized experimentally. For example, it is well-known that electrons above a liquid helium surface form a 2D electron layer. Now one can insert impurities in the substrate supporting the helium film, which may confine the electrons laterally. 


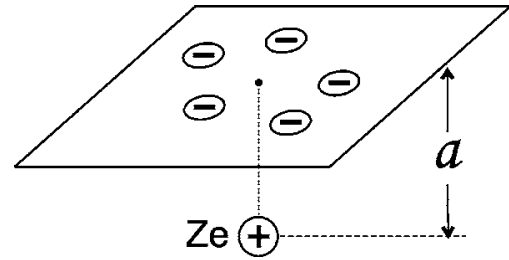

FIG. 1. Schematic view of the system.

Recently we studied the normal modes of a 2D Coulomb confined cluster of charged particles as a function of the strength of the confinement potential, and we found that the normal modes of the system are mainly controlled by the strength of the confinement potential [22]. In the present paper we investigate the thermal properties of the Coulomb bound cluster, i.e., the melting of the ordered configuration of particles and the thermal ionization process. Also very recently, we showed that temperature-induced phase transitions occur in the Coulomb bound cluster before the complete melting of the system takes place [27]. These observations were also extended to the case of small clusters of charged particles confined in a parabolic trap [28].

The present paper is organized as follows. In Sec. II we present the model of our numerical approach. The analysis of the melting in the present system is given in Sec. III. The phenomenon of evaporation (or ionization) is discussed in Sec. IV. Finally, our conclusions are given in Sec. V.

\section{NUMERICAL APPROACH}

We studied a system of $N$ negatively charged particles with charge $-e$, interacting through a repulsive Coulomb potential and moving in the $x y$-plane. The particles are kept together through a fixed positive charge $Z e$ located at a distance $a$ from the plane the particles are moving in (see Fig. 1). The total potential energy of this system is given by

$$
H=-\frac{Z e^{2}}{\epsilon} \sum_{i=1}^{N} \frac{1}{\sqrt{r_{i}^{2}+a^{2}}}+\frac{e^{2}}{\epsilon} \sum_{i>j=1}^{N} \frac{1}{\left|\mathbf{r}_{i}-\mathbf{r}_{j}\right|} .
$$

Here the symbol $\epsilon$ stands for the dielectric constant, and $\mathbf{r}$ $=\{x, y\}$ is the two component position vector of the particle. For convenience, we express the electron energy in units of $E_{0}=e^{2} /(\epsilon a)$ and all the distances in units of $a$. The temperature $T$ of the system will be expressed in units of $T_{0}$ $=E_{0} / k_{B}$, with $k_{B}$ the Boltzmann's constant. This allows us to rewrite Eq. (1) in the following dimensionless form:

$$
H=-\sum_{i=1}^{N} \frac{Z}{\sqrt{r_{i}^{2}+1}}+\sum_{i>j=1}^{N} \frac{1}{\left|\mathbf{r}_{i}-\mathbf{r}_{j}\right|} .
$$

Note that the potential energy only depends on two parameters, namely, the number of particles $N$ and the size of the positive confinement charge $Z$.

The ground state configurations of the two-dimensional system were obtained using the Monte Carlo (MC) method (using the standard Metropolis algorithm [29]) and the modified Newton method [20]. The negatively charged particles are initially put in random positions within some circular area and then allowed to reach a steady state configuration after about $10^{5}$ simulation steps. Simultaneously, we calculated the frequencies of the normal modes of the system using the Householder diagonalization technique [20]. The configuration was taken as final when all frequencies squared of the normal modes are positive and the energy did not decrease further.

To study melting the system was heated up (where temperature was increased by steps of $\delta T$, typically $\approx 10^{-3}$ ) and equilibrated at the new temperature during $5 \times 10^{4}$ to $10^{5}$ MC steps (one MC step corresponds with a displacement of all particles). After annealing, the average energy is calculated, together with the mean squared radial displacement

$$
\left\langle u_{R}^{2}\right\rangle \equiv \frac{1}{N} \sum_{i=1}^{N}\left(\left\langle r_{i}^{2}\right\rangle-\left\langle r_{i}\right\rangle^{2}\right) / \rho^{2},
$$

where $\rho$ is the average distance between the particles at zero temperature. The symbol \langle\rangle stands for an average over typically $10^{6} \mathrm{MC}$ steps after equilibration of the system.

\section{MELTING}

We studied the influence of thermal fluctuations on the configuration and investigate the structural phase transitions and melting in a Coulomb confined cluster. An important question is: does the confinement strength determine the melting temperature, or is the symmetry of the configuration more important? Preliminary results were presented in Ref. [27].

The behavior of the system is investigated in the soft confinement limit, i.e., $N \approx Z$, which corresponds to the (almost) neutral case, and where the correlational effects between the particles are much more pronounced [30]. The opposite limit, $N \ll Z$, is equivalent to the situation of a parabolic confined cluster, which was already studied in previous papers $[19,25]$. Here, the melting phenomenon is studied in small clusters $(N=12,11,10,9,8,7,6,5)$ for a fixed positive confinement charge $Z=12$. In order to characterize the melting temperature, we made use of a Lindemann-like criterion, which states that, in the case of Coulomb interaction, melting occurs when the mean radial displacement approaches the critical value $\left\langle u_{R}^{2}\right\rangle=0.05$ [19]. For finite size systems this criterion is not exact because we have now a nonzero temperature range over which melting occurs.

In Fig. 2, the mean squared radial displacement is presented as a function of temperature for clusters with $N$ $=12,11,10,9,8,7,6,5$ particles for fixed $Z=12$. The corresponding zero temperature ground state configurations are shown in Fig. 3. With exception the cluster $N=7$, metastable states were not observed in these cases. Intuitively one expects that the neutral system $(N=Z=12)$, which has the smallest confinement energy per particle, has the lowest melting temperature as can indeed be inferred from Fig. 2, while a higher melting temperature is expected with decreasing $N$ because the clusters become more strongly confined. Surprisingly, the latter behavior is not found in small clusters. Figure 2 shows that the cluster $(N=11 ; Z=12)$ has a higher melting temperature than the clusters $(N=10,9,8,7$; 


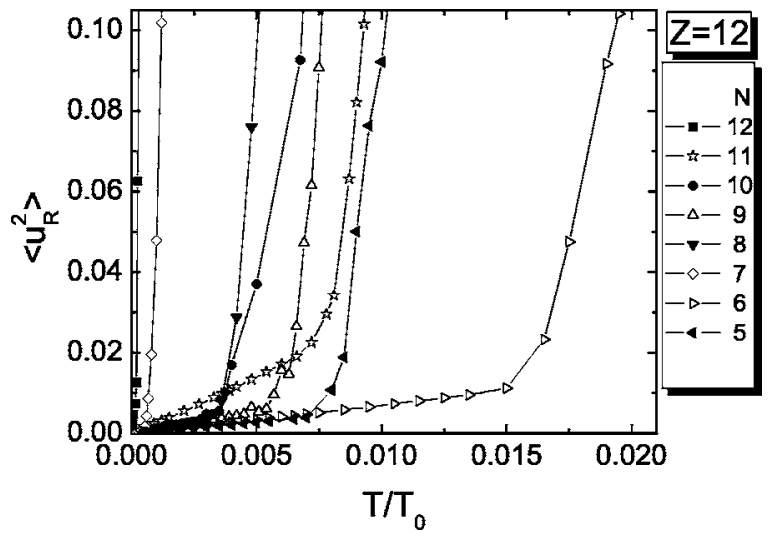

FIG. 2. The squared mean radial deviation as a function of temperature, for clusters with different number of particles, which are confined by a positive charge $Z=12$.

$Z=12$ ), which have higher net charge. Thus the confinement energy is not the only determining factor for the value of the melting temperature. In order to understand this, we investigated the symmetry of the ground state configuration of the different clusters. To characterize the symmetry of a configuration, the different rotational symmetries which leave the configuration invariant are considered. A rotation operator over an angle $2 \pi / n$ is indicated as $C_{n}$, and the larger the value of $n$ the higher the symmetry of the configuration.

In spite of the lower confinement energy per particle, the cluster $(N=11 ; Z=12)$, which is invariant under rotation $C_{5}$, has a higher melting temperature than the less symmetric clusters $\quad(N=10,9,8,7 ; \quad Z=12) \quad$ (invariant under $C_{2}, C_{3}, C_{2}, C_{3}$, respectively). This fact made us conclude that the more packed and the more symmetric the particles are arranged, the larger the stability, and consequently, the larger the melting temperature. This statement is confirmed by the highly symmetric cluster $(N=6 ; Z=12)$ which has the largest melting temperature (see Fig. 2). Note that its melting temperature is even higher than the one for the cluster $(N=5$; $Z=12$ ). When comparing the configurations of the clusters $N=5$ (invariant under $C_{4}$ ) and $N=6$ (invariant under $C_{5}$ ), we observe that the last one is more symmetric. A similar dependence of the value of the melting temperature on the sym-
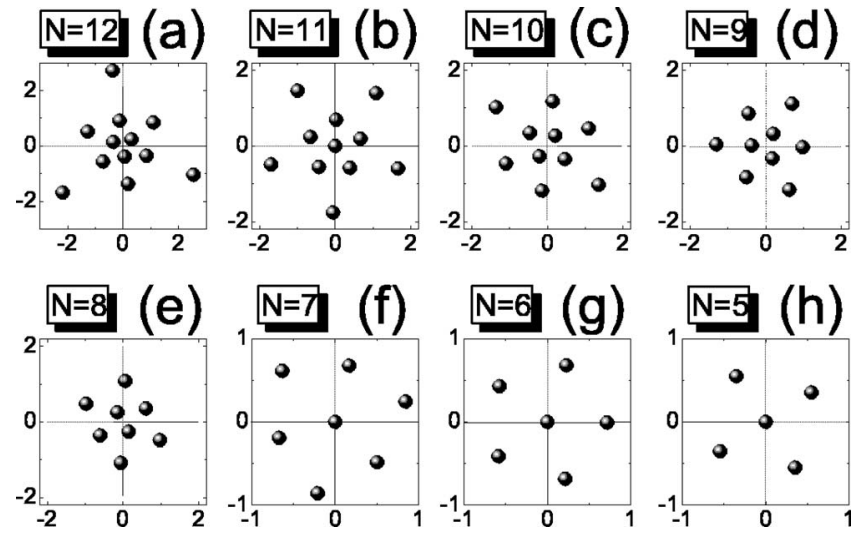

FIG. 3. Ground state configurations for $T=0$. All clusters are confined by a positive charge $Z=12$.

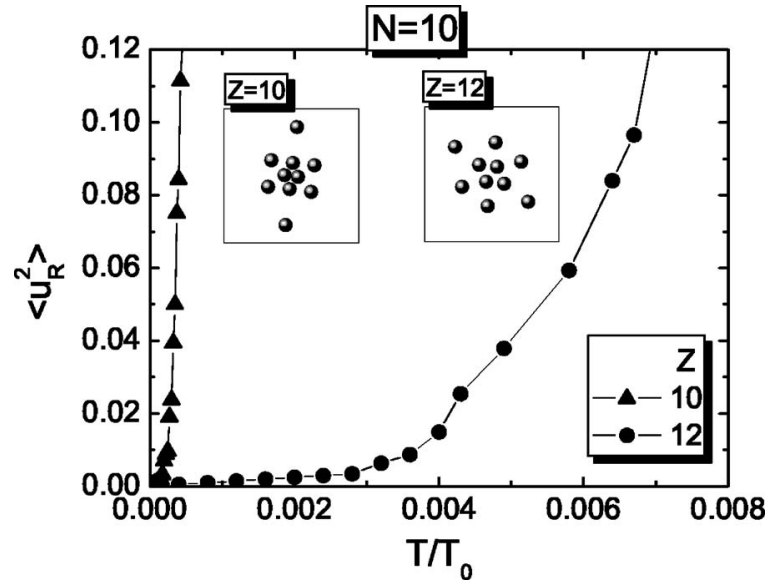

FIG. 4. The mean squared radial displacement as a function of temperature, for clusters consisting of $N=10$ particles, confined by positive charges $Z=10$ and $Z=12$. The corresponding ground state configurations at $T=0$ are shown as insets.

metry of the cluster was also observed in other clusters with different values for $N$ and $Z$. But in cases when the symmetry of the configurations is the same, the melting temperature is determined by the strength of the confinement potential, as shown in Fig. 4 . The system $N=10 ; Z=12$ is more strongly confined than the system $N=Z=10$, and it has indeed a higher melting temperature while they have the same symmetry as clearly indicated in Fig. 4. From the previous results, we can conclude that the symmetry of the ground state configuration is the dominant factor which determines the melting temperature in small Coulomb confined clusters.

Next we investigated the effect of the presence of metastable states on the melting temperature. As a typical example, we consider a cluster with $N=7$ particles, which we submit to different strengths of the confinement potential. In Fig. 5, we show the corresponding ground state configurations. Metastable states were observed only in the interval $11.3 \leqq Z<13.5$, and the corresponding configurations are also presented in Fig. 5. For $Z \lesssim 11.9$, the ground state configurations have approximately the same symmetry (with a rotation axis $C_{1}$ ), as clearly can be seen in Fig. 5. The same is seen for $Z>11.9$, but now the new configurations have a higher symmetry, i.e., $C_{3}$ [Figs. 5(i), 5(k), 5(m), and 5(o)].

The melting temperature as a function of $Z$ for the cluster with $N=7$ particles is shown in Fig. 6. We define regions (I) $[Z \lesssim 11.9)]$ and (II) $[Z>11.9)]$, where the ground state configurations are different (see inset in Fig. 6). Starting from the neutral case $(Z=N=7)$, we notice that the melting temperature increases with increasing strength of the confinement potential up to $Z=10$, which is in accordance of the above conclusions that for clusters with identical symmetry the strength of the confinement determines the melting temperature. For $Z>10$, the melting temperature decreases with increasing $Z$, up to $Z \approx 12$. In the interval $11.3 \lesssim Z<13.5$ the system has metastable states, and when $Z \rightarrow 11.9$ the difference between the energy of the ground state and the energy of the metastable state $\left(E_{M S}-E_{G}\right)$ becomes smaller (see open symbols in Fig. 6). The height of the saddle point between the two local minima should also become smaller (Fig. 7). 

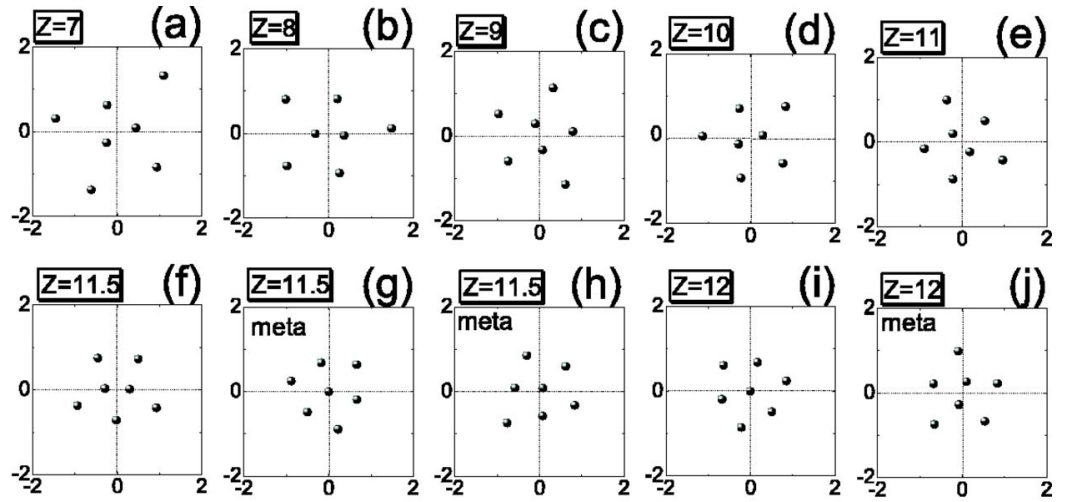

FIG. 5. (Color online) Ground state configurations for the cluster $N=7$, submitted to different confinement strengths $Z$.
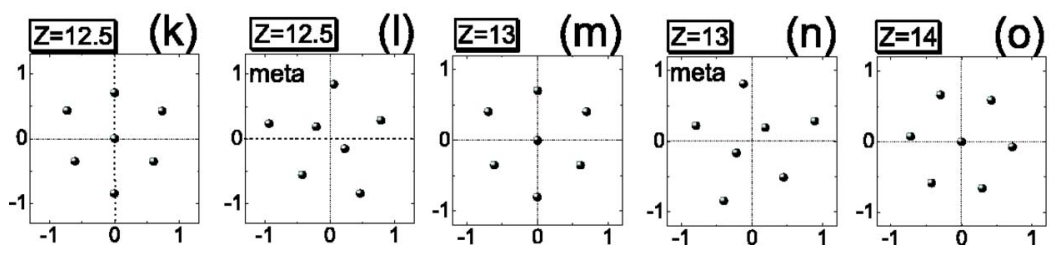

As consequence, less thermal energy is needed for the cluster to transit from one configuration to the other and thus the melting temperature clearly follows the energy difference between the ground state and the lowest metastable state. For $Z>11.9$, the symmetry of the ground state configuration increases [see inset in Fig. 6-region(II)]. The difference $\left(E_{M S}-E_{G}\right)$ becomes larger with increasing value of $Z$. For $Z>13.5$, no metastable states are found. The symmetry of the ground state configuration is larger and consequently also its melting temperature.

The same behavior of the melting temperature is observed for clusters with a different number of particles, as can be seen, e.g., in Fig. 8, for the cluster with $N=11$ particles. We define again two regions, according to the symmetry of the ground state configuration, which are shown as insets in Fig. 8 . For $Z \leqslant 16.7$ the ground state configuration is like the one presented in region (I), while for $Z>16.7$ the system changes to the configuration shown in region (II). Metastable states appear only for $Z \geqslant 15.4$. As the previous case for the cluster with $N=7$ particles, the melting temperature exhibits

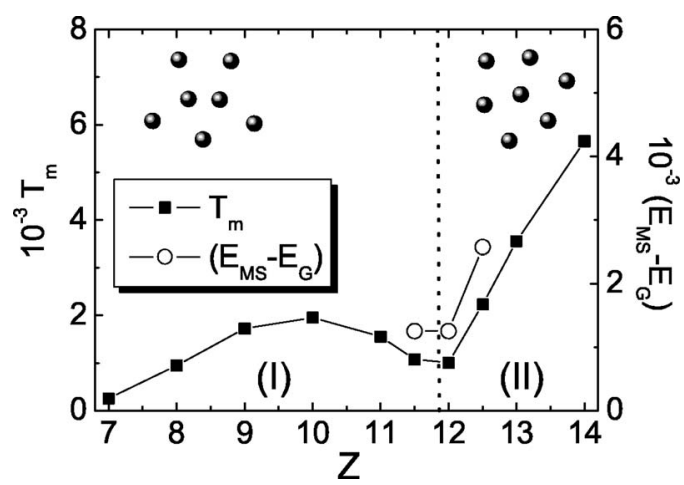

FIG. 6. The melting temperature as a function of $Z$ for the cluster with $N=7$ particles (closed squares). The difference between the energy of the metastable state $\left(E_{M S}\right)$ and the energy of the ground state $\left(E_{G}\right)$ is also shown (open circles) with reference to the right axis. a minimum in the $Z$-interval where the ground state configuration switches from one configuration to another, and where metastable states are observed. Recently, Kong et al. also found that the melting temperature of a $2 \mathrm{D}$ parabolic confined cluster of particles interacting through a Yukawa potential is determined by the saddle point energy [25].

\section{EVAPORATION}

In the present system the confinement potential reduces to zero at infinity which allows for an unbinding of charges,

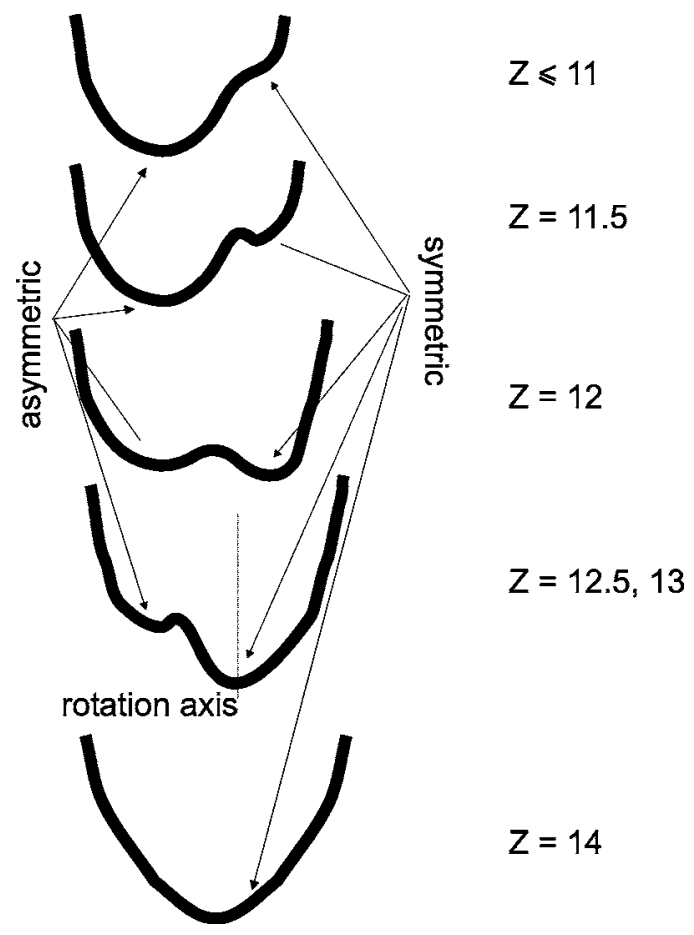

FIG. 7. Sketch of the energy landscape for the cluster with $N$ $=7$ particles under different confinement strengths. 


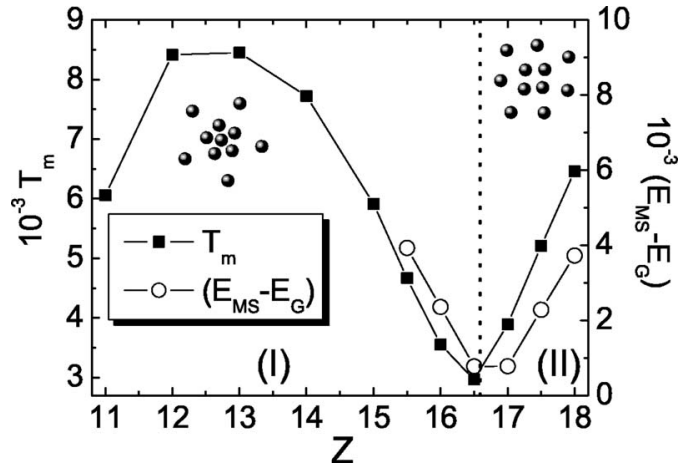

FIG. 8. The same as Fig. 6, but now for $N=11$.

i.e., ionization of the cluster or evaporation [26,31]. For nonzero temperature one particle may lend sufficient thermal energy in order to get a large enough velocity to become unbound. The difference in energy between the clusters with $N$ and $N-1$ particles is the binding energy of one particle to the cluster with $N$ particles. In this section, we analyze the Coulomb bound cluster for temperatures beyond the melting temperature $T_{m}$. To do so, it is convenient to define some quantities as, e.g., the mean density, $\rho(r)$, and the mean pair distribution function, $g(r)$. In our simulations, these quantities were averaged over all Monte Carlo steps (typically 3 $\times 10^{7}$ steps).

As a representative example, we consider the cluster with $N=10(Z=10)$ particles. In Figs. 9(a) and 9(b) the quantities $\rho(r)$ and $g(r)$ are shown as a function of temperature. Notice that the temperature dependence of $\rho(r)$ and $g(r)$ indicate a nonuniform melting of the system, i.e., the external shells melt before the internal ones. This phenomenon is due to the nature of the confinement potential. The interaction between the confinement charge and the external particles is screened by the particles in the central region of the cluster, resulting in a weaker effective confinement potential for the edge particles. Melting occurs at $T_{m} \approx 0.0012$, and a further increase of the temperature leads eventually to the possibility of evaporation of the first particle (at $T / T_{0} \geq 0.018$ ). This can be seen in the inset of Fig. 9(a), which shows that the mean radial displacement increases very fast with increasing temperature for large $T$. The cluster now has $N=9$ particles, and its net positive charge is +1 , which implies a higher effective confinement energy per particle. The $g(r)$ curves show that, in the temperature interval corresponding to the unbinding of one particle, the system is clearly in the liquid state with only short range order [see Fig. 9(b), $T / T_{0}=0.017$ ]. The mean density curve at this temperature also reveals a diluted system with no apparent structure. For higher temperatures (see the curve for $\left.T / T_{0}=0.030\right)$ a broad peak in the mean density curve is formed around $r \approx 0.4$. This indicates a reorganization of the particles in the system, where now three particles are situated on the first ring with smaller radial oscillations than the other ones located at larger distances from the center. This conclusion is supported by the results of the mean density and the mean pair distribution function for the cluster $(N=9 ; Z=10)$ at temperatures larger than the evaporation temperature which corresponds to the ones observed in the cluster $(N=10 ; Z=10)$, at the same temperatures.
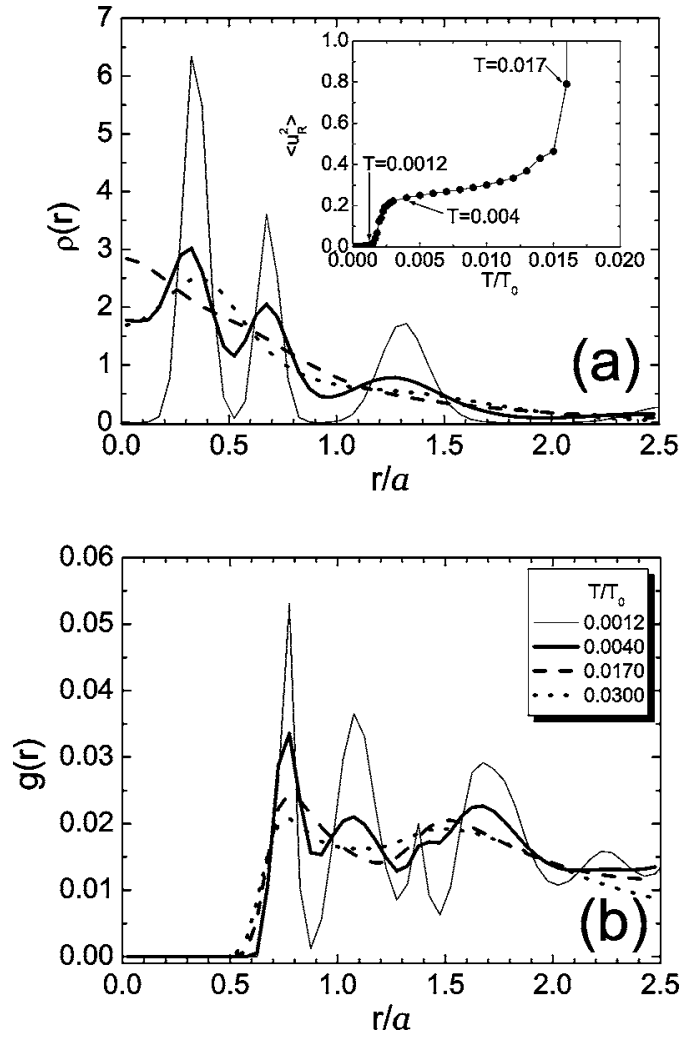

FIG. 9. The density (a) and the pair distribution (b) function $g(r)$ as a function of distance, for the cluster with $(N=10, Z=10)$ at different temperatures. The inset of (a) shows the average radial displacement as a function of temperature.

The evaporation of particles in the present system can be nicely illustrated by considering the caloric curves, i.e., the energy as a function of temperature. The caloric curves for the clusters $(N=10,9,8,7,6 ; Z=10)$ are shown in Fig. 10. A precise evaporation temperature for a single particle cannot be obtained due to the statistical nature of the evaporation process. Different Monte Carlo runs lead to different times for evaporation of the particle at a given temperature. For this reason we averaged all the calculated quantities, i.e., the values of energy, over an ensemble of Monte Carlo runs with

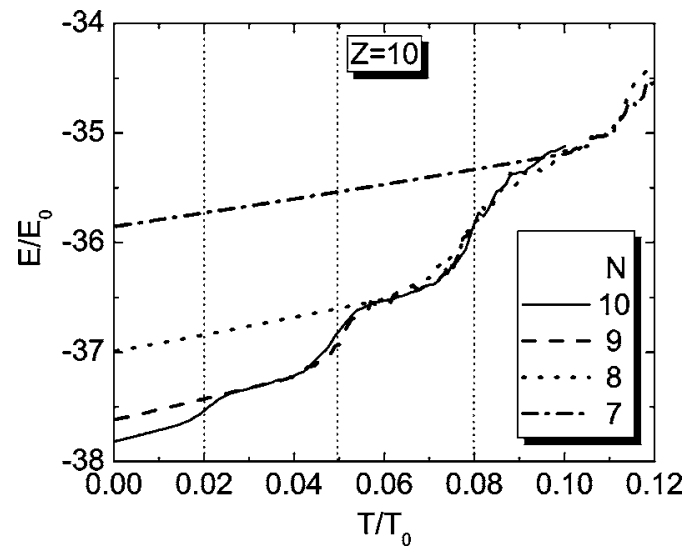

FIG. 10. The energy as a function of temperature for clusters confined by a positive charge $Z=10$. 
fixed duration. Due to this statistical averaging procedure and the finite size of the considered system, the evaporation process is smeared out, and we define the evaporation temperature $\left(T_{e v}\right)$, which characterizes the evaporation of a single charged particle from the $N$ particle system, as the temperature at which the second derivative of the energy with respect to $T$ is zero [32]. Such a temperature corresponds to the midpoint of the transition region in the $E$ versus $T$ curve. The evaporation temperature is indicated by the vertical thin dotted lines in Fig. 10. As can be observed, the energy curve for the cluster with $N=10$ particles (solid line in Fig. 10) merges into the one for the cluster with $N=9$ particles (dashed line in Fig. 10) after the unbinding of the first particle. In the same way, the curve for the cluster $N$ $=9$ merges into the one for the cluster $N=8$ after the evaporation of one more particle at $T_{e v} / T_{0} \approx 0.05$. For higher temperatures the evaporation of the other particles occurs, one by one, and a similar behavior is observed in the caloric curve as a function of $T$.

From Fig. 10 we notice that the binding energy increases with increasing net charge of the system. Such a behavior is clear since the particles become more and more confined. The increase in the confinement energy of the particles is reflected directly in the rate at which the energy is absorbed by the system as a function of temperature. The ratio between the absorbed energy and the variation in the temperature of the system is the so-called heat capacity $C$. In the present system, the heat capacity was obtained from the linear part of the caloric curves, before the evaporation of a particle takes place, by calculating the slope of such a linear curve. A high $C$ means a high absorption rate of energy with increasing temperature. The heat capacity for the clusters with $N=10,9,8,7,6,5,4,3$ (all of them confined by a positive charge $Z=10$ ) are presented in Fig. 11. The heat capacity decreases with decreasing number of particles. Two different regimes can be distinguished, related to two different configurational types. In the interval $7 \leqslant N \leqslant 10$ the increase in $C$ is larger than the one in the interval $3 \leqslant N \leqslant 6$, in which the configuration of the clusters corresponds to particles placed on a single ring (for $N=5$ and $N=6$ there is also one particle in the center).

\section{CONCLUSIONS}

We studied the thermal properties of a 2D classical system of charges in a nonuniform charged background. We ad-

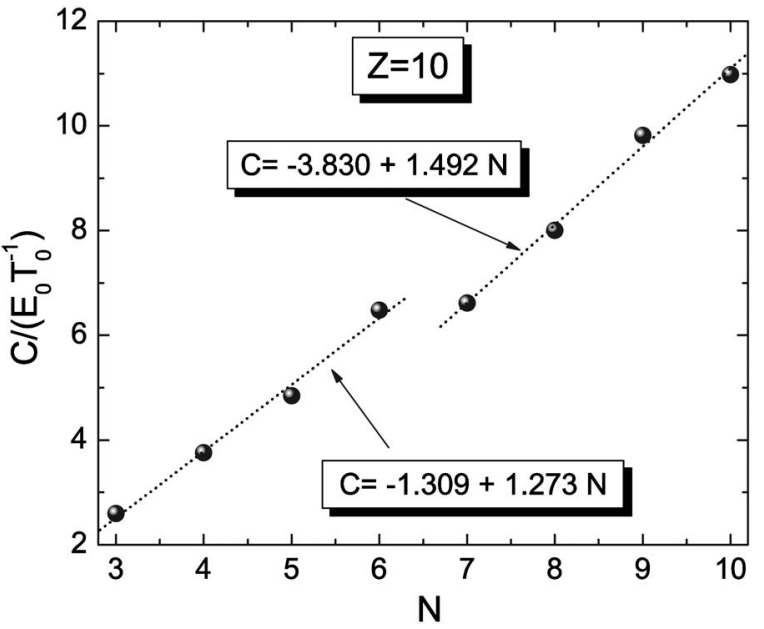

FIG. 11. The heat capacity for clusters with different number of particles $N$ confined by a positive charge $Z=10$.

dressed melting in clusters with a small number of particles. The considered nonuniform background introduces a new property, namely, the evaporation of particles, which is not observed in the parabolic confinement case. The solid-liquid transition in Coulomb bound clusters is altered by the presence of metastable states. When such metastable states are present, we showed that the melting temperature can be strongly affected and is now determined by the height of the saddle point energy separating the lowest metastable state and the ground state. After the melting transition, the Coulomb bound cluster can become ionized and particles unbind. Due to the finite size of the system the evaporation of particles occurs one by one, which is a statistical process. The latter results lead to a temperature evaporation region. The importance of the symmetry of the ground state configuration on the melting phenomenon was also shown for different cases of those ones presented in our previous work [27]. The higher the symmetry of the configuration the higher the melting temperature. However, when the systems have the same configuration, the one with stronger confinement has a higher melting temperature.

\section{ACKNOWLEDGMENTS}

G.A.F. was supported by the Brazilian National Research Councils (CNPq, CAPES), and the Ministry of Planning (FINEP). Part of this work was supported by the Flemish Science Foundation (FWO-Vl). We thank B. Partoens for helpful discussions.
[1] R. S. Crandall and R. Williams, Phys. Lett. 34, 404 (1971).

[2] C. C. Grimes and G. Adams, Phys. Rev. Lett. 42, 795 (1979).

[3] M. Golosovsky, Y. Saado, and D. Davidov, Phys. Rev. E 65, 061405 (2002).

[4] J. H. Chu and L. I, Phys. Rev. Lett. 72, 4009 (1994).

[5] L. Jacak, P. Hawrylak, and A. Wójs, Quantum Dots (SpringerVerlag, Berlin, 1998).

[6] M. Saint Jean, C. Even, and C. Guthmann, Europhys. Lett. 55,
45 (2001)

[7] B. A. Grzybowski, H. A. Stone, and G. M. Whitesides, Nature (London) 405, 1033 (2000).

[8] B. A. Grzybowski, X. Jiang, H. A. Stone, and G. M. Whitesides, Phys. Rev. E 64, 011603 (2001).

[9] E. Wigner, Phys. Rev. 46, 1002 (1934).

[10] J. M. Kosterlitz and D. J. Thouless, J. Phys. C 6, 1181 (1973).

[11] B. I. Halperin and D. R. Nelson, Phys. Rev. Lett. 41, 121 
(1978).

[12] A. P. Young, Phys. Rev. B 19, 1855 (1979).

[13] A. Melzer, Phys. Rev. E 67, 016411 (2003).

[14] R. Bubeck, C. Bechinger, S. Neser, and P. Leiderer, Phys. Rev. Lett. 82, 3364 (1999).

[15] K. Zahn, R. Lenke, and G. Maret, Phys. Rev. Lett. 82, 2721 (1999).

[16] P. S. Branício, J. P. Rino, and N. Studart, Phys. Rev. B 64, 193413 (2001).

[17] Weijia Wen, Lingyun Zhang, and Ping Sheng, Phys. Rev. Lett. 85, 5464 (2000).

[18] D. B. Mast, A. J. Dahm, and A. L. Fetter, Phys. Rev. Lett. 54, 1706 (1985).

[19] V. M. Bedanov and F. M. Peeters, Phys. Rev. B 49, 2667 (1994).

[20] V. A. Schweigert and F. M. Peeters, Phys. Rev. B 51, 7700 (1995).

[21] V. A. Schweigert and F. M. Peeters, J. Phys.: Condens. Matter 10, 2417 (1998)

[22] W. P. Ferreira, F. M. Peeters, and G. A. Farias, Phys. Rev. E
68, 066405 (2003).

[23] A. V. Filinov, M. Bonitz, and Yu. E. Lozovik, Phys. Rev. Lett. 86, 3851 (2001).

[24] I. V. Schweigert, V. A. Schweigert, and F. M. Peeters, Phys. Rev. Lett. 84, 4381 (2000).

[25] M. Kong, B. Partoens, and F. M. Peeters, New J. Phys. 5, 23 (2003).

[26] G. A. Farias and F. M. Peeters, Solid State Commun. 100, 711 (1996).

[27] W. P. Ferreira, B. Partoens, F. M. Peeters, and G. A. Farias, Phys. Rev. E 71, 021501 (2005).

[28] D. Tomecka, B. Partoens, and F. M. Peeters, Phys. Rev. E 71, 062401 (2005)

[29] N. Metropolis, A. W. Rosenbluth, M. N. Rosenbluth, A. M. Teller, and E. Teller, J. Chem. Phys. 21, 1087 (1953).

[30] W. P. Ferreira, A. Matulis, G. A. Farias, and F. M. Peeters, Phys. Rev. E 67, 046601 (2003).

[31] W. P. Ferreira, G. A. Farias, H. A. Carmona, and F. M. Peeters, Solid State Commun. 122, 665 (2002).

[32] M. Bixon and Joshua Jortner, J. Chem. Phys. 91, 1632 (1989). 